\title{
Bio-inspired hair-based inertial sensors
}

\author{
H. Droogendijk, M. J. de Boer, R. G. P. Sanders and G. J. M. Krijnen \\ $\mathrm{MESA}^{+}$Institute for Nanotechnology, University of Twente, Enschede, THE \\ NETHERLANDS \\ E-mail: h.droogendijk@utwente.nl
}

Submitted to: Biomimetic technologies

\begin{abstract}
In biology, hair-based sensor systems are used regularly for measurement of physical quantities like acceleration, flow, rotational rate and IR-light. In this chapter, two different types of bio-inspired sensors for inertial measurement are discussed, which have been developed using surface micromachining and SU-8 lithography. First, an accelerometer inspired by the cricket's clavate hair is presented. Second, a gyroscope inspired by the fly's haltere is treated. For both sensors are the necessary models presented, and guidelines are derived for optimization. Also, their performance is compared to their biological counterpart and the biomimetic potential is discussed.
\end{abstract}




\section{Contents}

1 Introduction $\quad 2$

2 Hair structures for inertial sensing $\quad 3$

2.1 Cricket's clavate hairs . . . . . . . . . . . . . . . . . . 3

2.2 Fly's haltere ............................ 4

3 Cricket-inspired accelerometer $\quad 5$

3.1 Physics . . . . . . . . . . . . . . . . . . 5

3.2 Design . . . . . . . . . . . . . . . . . 7

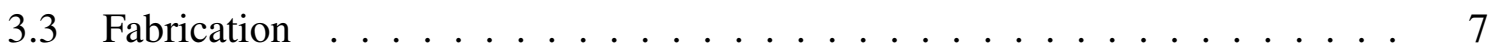

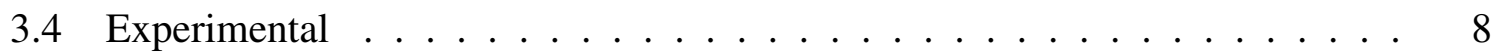

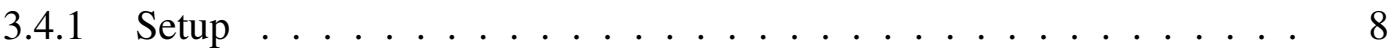

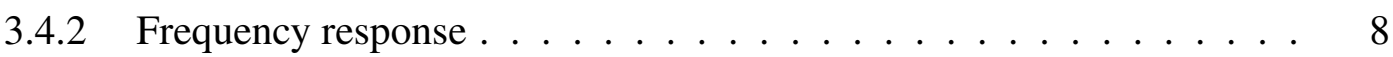

3.4.3 Directivity ......................... 9

3.4.4 Threshold and linearity . . . . . . . . . . . . . . . . 10

3.5 Discussion . . . . . . . . . . . . . . . . . . 11

4 Fly-inspired gyroscope $\quad 13$

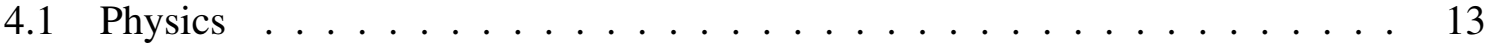

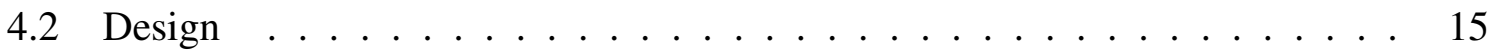

4.3 Fabrication . . . . . . . . . . . . . . . . . 19

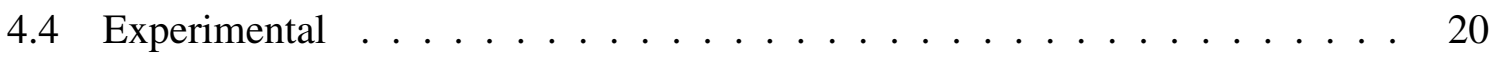

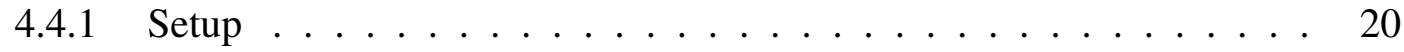

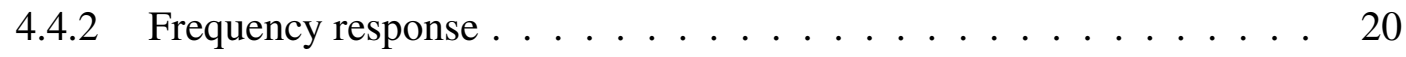

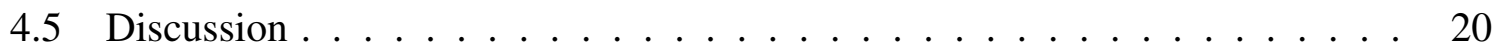

5 Bio-inspiration continued $\quad 22$

6 Conclusions $\quad 22$

\section{Introduction}

In biology, mechano sensors, equipped with differing hair-like structures for signal pick-up, are sensitive to a variety of physical quantities like acceleration, flow, rotational rate, balancing and IR-light (Liu, 2007; Schmitz et al., 2012). As an example, crickets have various types of hair-like receptors for measurement of several environmental quantities. For sensing of low-frequency flows (typically $<1 \mathrm{kHz}$ ) to obtain information about the environment and avoid e.g. predator attacks crickets use filiform hairs, which are situated on the dorsal side of two abdominal appendages called cerci, and which are able to sense airflows with velocity amplitudes down to $30 \mu^{-1} \mathrm{~s}^{-1}$ (Tobias and Murphey, 1979; Shimozawa and Kanou, 1984; Shimozawa et al., 1998; Humphrey et al., 1993) and operate around the energy levels 
of thermal noise (Shimozawa et al., 2003). Crickets gather also information about their environment by the use of bristle hairs which activate interneurons that respond to tactile stimuli of the cercus and abdomen (Murphey, 1985).

It are these intriguing aspects of biological sensors that inspire engineers on developing artificial counterparts and exploiting the field of biomimetics and bio-inspiration. For example, taking the cricket cercal hair sensors as a source of inspiration, several research groups have worked on the development of bio-inspired hair-based airflow measurements by exploiting microelectromechanical systems (MEMS) technology (Ozaki et al., 2000; Chen et al., 2007; Wang et al., 2007; Sadeghi et al., 2011; Tao and Yu, 2012).

In this chapter, the bio-inspired approach is applied to bio-inspired inertial sensors developed using MEMS-technology. Particularly, two types of bio-inspired inertial sensors are discussed: a cricket-inspired accelerometer (Droogendijk et al., 2014b) and a fly-inspired gyroscope (Droogendijk et al., 2014a). The main outline of this chapter is as follows. Section 2 deals with two examples of biological sensor systems for measurement of inertial quantities. Subsequently, the design and fabrication of the cricket-inspired accelerometer (section 3) and the fly-inspired gyroscope (section 4) are discussed. Section 5 continues with the assessment of the bio-inspired approach within the field of (inertial) hair-based sensors.

\section{Hair structures for inertial sensing}

\subsection{Cricket's clavate hairs}

In addition to flow and tactile perception, crickets have club-shaped sensilla, called clavate hairs, located on their cerci (figure 1), with hair lengths of 20-250 $\mu \mathrm{m}$ (Bischof, 1974). These clavate hairs turn out to be sensitive to (gravitational) acceleration, providing the cricket information on its orientation (Bischof, 1974, 1975; Murphey, 1981; Sakaguchi and Murphey, 1983). For example, a cricket uses its clavate hairs to compensate head movement when it is rotated around its longitudinal axis (Horn and Bischof, 1983), for which such rotations can be measured with a resolution of about $0.1^{\circ}$ (Bischof, 1975). Additionally, Bischof (1975) showed that these clavate hairs can respond to harmonic accelerations with frequencies up to $300 \mathrm{~Hz}$

For measuring (gravitational) acceleration, numerous types of accelerometers have been realized over the past years using MEMS technology, with applications in e.g. the automotive industry and navigation (Yazdi et al., 1998). Current state-of-the-art commercialized MEMS accelerometers show formidable performance in range, resolution and noise floor. In contrast to the cricket's clavate system, MEMS accelerometers are usually not hair-based systems and frequently contain feedback electronics. To explore some of the intricacies of the clavate hair system and assess its potential to engineering applications (e.g. automotive industry, robotics and motion tracking), we aim for the design, fabrication and characterization of a bio-inspired accelerometer. Bio-inspired hair-based structures have been exploited earlier with applications in both actuation and sensing of physical quantities (Zhou and Liu, 2008; Ginsberg et al., 2013), but seldom for inertial measurement. Previously, a hair- 


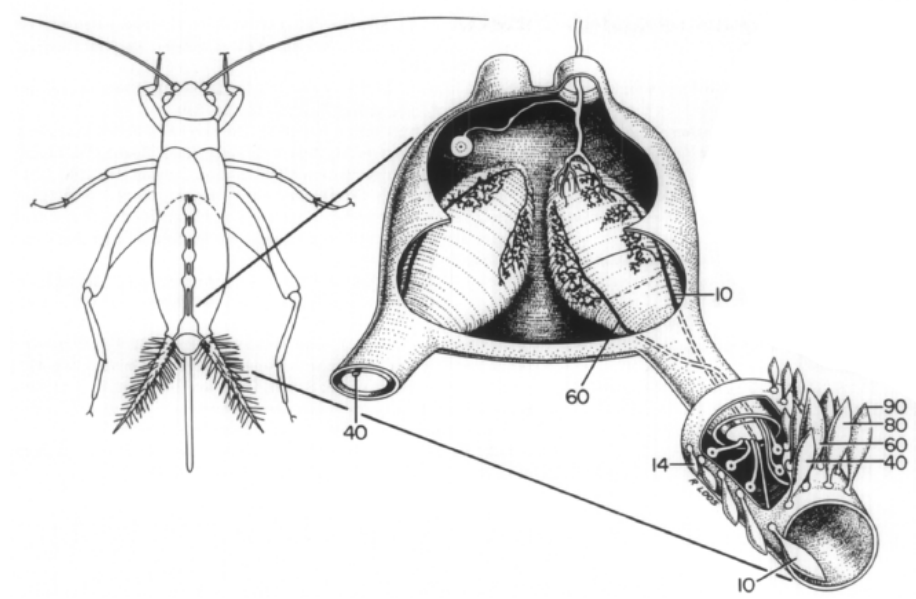

Figure 1. Artist's reconstruction of the clavate hair-based sensory system (adapted from (Murphey, 1981)).

like accelerometer has been investigated by Tang et al. (2013), but its response to external accelerations was not demonstrated.

\subsection{Fly's haltere}

Another example of a biological hair-based sensory system is the fly's haltere structure. Halteres are tiny club-shaped organs that beat in anti-phase to the wings during flight (Fraenkel and Pringle, 1938), and function as gyroscopes $\neq$ by measuring the flies body rotation using Coriolis forces (Pringle, 1948). The rotation-dependent position of the haltere (figure 2) is measured in sensitive strain fields (Hengstenberg, 1998). It has been demonstrated that this haltere-based system is used for supporting equilibrium reflexes (Sandeman and Markl, 1980; Nalbach and Hengstenberg, 1994; Chan et al., 1998; Ristroph et al., 2010).

For measuring angular rate, numerous types of gyroscopes have been realized over the past years using MEMS technology, where current state-of-the-art MEMS gyroscopes show formidable performance in range, resolution and noise floor (Yazdi et al., 1998; Xie and Fedder, 2003; Alper and Akin, 2005; Trusov et al., 2011). In contrast to the fly's haltere system, MEMS gyroscopes are typically no hair-based systems, are generally heavily underdamped and operate at rather high frequencies. In contrast, the fly's haltere system is a close to critically damped system and operates around the fly's wing beat frequency (130-150Hz) (Nalbach and Hengstenberg, 1994). Obviously, the fly's haltere system has developed under evolutionary pressure and therefore addresses the sensory needs of the fly. As a result, not only the operation principle is different from those generally employed in MEMS gyroscopes but also the performance may score high on metrics other than normally used for MEMS gyroscopes. For example, bandwidth and response time may be far more essential for flies than (bias-)stability and noise floor, as the halteres have been proven to aid

$\$$ In this work, all gyroscopes are so-called Coriolis vibratory gyroscopes, which are in fact angular rate sensors. By integration of the measured angular rate, information about the angle is obtained. 


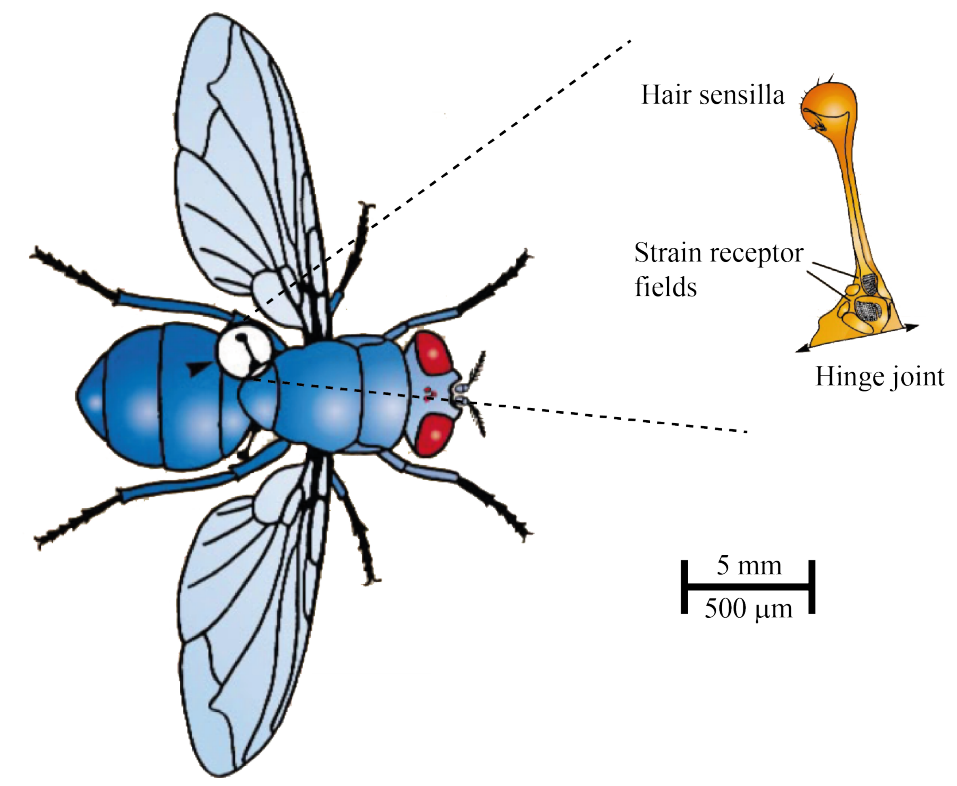

Figure 2. Halteres of the blowfly Calliphora vicina. During walking and flight, the halteres oscillate in a vertical plane around a proximal hinge (adapted from (Hengstenberg, 1998)).

in flight dynamics of flies with fast (10ms) responses to flight disturbances (Rosner et al., 2009).

Previous research on bio-inspired gyroscopic systems (using foils of stainless steel) shows advantages on using a haltere-based gyroscope by achieving a higher sensitivity and a lower power consumption compared to conventional MEMS gyroscopes (Wu et al., 2002; Wu and Wood, 2006). Also Tang et al. (1997) and Challoner et al. (2007) fabricated hairbased gyroscopes using MEMS technology, although these devices had still macroscopic dimensions compared to the fly's haltere and were severely underdamped by operating them in vacuum. Wicaksono et al. (2007) discuss the preliminary design and modelling of a bioinspired MEMS gyroscope with electrostatic driving and piezo-resistive read-out. Smith et al. (2012) report on the design and fabrication of a MEMS haltere to use as a sensor in a microautonomous system.

\section{Cricket-inspired accelerometer}

\subsection{Physics}

Mechanically, the hair-based accelerometer can be understood as a so-called inverted pendulum which is subjected to external accelerations (figure 3). It is described as a secondorder rotational-mechanical system with moment of inertia $J$, a rotational stiffness $S$ and a rotational damping $R$, resulting in a description of the system's response to harmonic accelerations by

$$
\theta(\omega)=\frac{T_{\mathrm{a}}}{S-J \omega^{2}+j \omega R},
$$




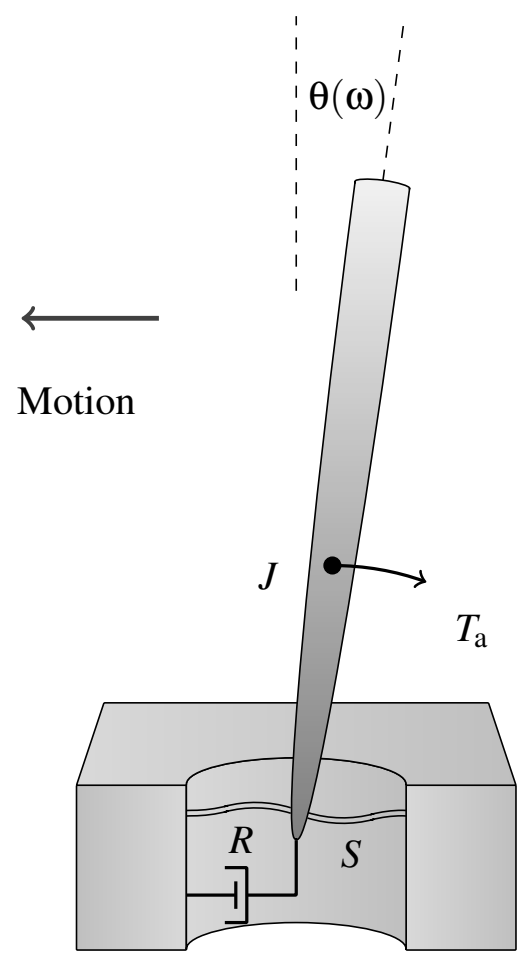

Figure 3. Schematic view of inertial measurement by hair-like structures.

where for a cylindrical hair, the moment of inertia can be expressed as

$$
J=\frac{\pi \rho d^{2}}{48}\left(4 L^{3}+\frac{3}{4} d^{2} L\right)
$$

In these expressions, $\theta(\omega)$ is the rotational angle amplitude of the hair and $T_{\mathrm{a}}$ is the amplitude of the torque acting on the hair. Usually, in the models the hairs are treated as cylindrical structures (Humphrey et al., 1993), but here the hair is modelled as an inverted conical shape to take the MEMS-hair-shape into account. We will show that this eventually will only lead to a geometry dependent factor $\eta$ such that $J \approx \eta \rho d^{2} L^{3}$.

From (2), the moment of inertia $J$ depends strongly on the hair diameter $d$ and hair length $L$. The torque $T_{\mathrm{a}}$ is a consequence of external accelerations, or the projections thereof, in the direction perpendicular to the hair and perpendicular to its rotation axis, denoted here by $a_{\text {ext }}$. Using Newton's second law $T_{\mathrm{a}}$ is found by integrating the inertial contributions by over the hair:

$$
T_{\mathrm{a}}=\int_{0}^{L} a_{\mathrm{ext}} \rho \pi\left(\frac{d(z)}{2}\right)^{2} z \mathrm{~d} z=\Xi \cdot a_{\mathrm{ext}},
$$

where $\Xi$ can be expressed as

$$
\Xi=\frac{\pi \eta \rho}{8} d^{2} L^{2} .
$$


In these expressions, $\rho$ is the density of the hair and $\eta$ a parameter that depends on the precise geometry of the hair. Further, we assume that the rotational angle amplitudes $\theta$ are small, so the torque $T_{\mathrm{a}}$ can be considered directly proportional to the external acceleration $a_{\text {ext }}$.

\subsection{Design}

The hair mechanical system behaves like a classical-second order system, and consequently exhibits the trade-off between responsivity and bandwidth. The responsivity of the hair accelerometer for frequencies well below the system's resonance frequency $\left(\omega \ll \omega_{\mathrm{r}}\right)$ is defined as

$$
\text { Responsivity }=\left.\frac{\mathrm{d} \theta(\omega)}{\mathrm{d} a_{\mathrm{ext}}}\right|_{\omega=0}=\frac{\pi \eta}{8} \frac{\rho d^{2} L^{2}}{S} .
$$

The bandwidth of the system is estimated from the system's resonance frequency:

$$
\text { Bandwidth }=\omega_{\mathrm{r}}=\sqrt{\frac{S}{J}} .
$$

By taking the product of responsivity and bandwidth, a figure of merit (FoM) can be defined for the bio-inspired hair accelerometer, similar to the approach described by Krijnen et al. (2007):

$$
\text { FoM }=\text { Responsivity } \times \text { Bandwidth. }
$$

When the hair length is considerably larger than the hair diameter $(L \gg d)$, this FoM can be simplified to

$$
\text { FoM } \propto \sqrt{\frac{\rho d^{2} L}{S}} .
$$

As a result, to achieve a 'good' hair-based accelerometer, the sensor should have a long and thick hair (high $d$ and $L$ ), as well as a compliant mechanical suspension (low $S$ ).

\subsection{Fabrication}

The fabrication process for the bio-inspired accelerometer is based upon the process for cricket-inspired bio-inspired hair flow sensors, previously developed in our group (Bruinink et al., 2009; Dagamseh et al., 2011). A schematic overview of the bio-inspired accelerometer with the materials indicated is shown in figure $4 \mathrm{a}$.

The sensor is fabricated on a silicon-on-insulator wafer. Trenches are etched in the silicon device layer using DRIE (deep reactive-ion etching). A layer of $200 \mathrm{~nm}$ stoichiometric $\mathrm{Si}_{3} \mathrm{~N}_{4}$ is used for covering and protecting the trenches. The device layer contains two electrodes, which are used for capacitive readout of the acceleration-induced movement. On top of the $\mathrm{Si}_{3} \mathrm{~N}_{4}$ layer, a sacrificial layer of poly-silicon $(1.5 \mu \mathrm{m})$ is deposited by LPCVD (low-pressure chemical vapour deposition). The sensor membrane and springs are constructed by depositing and patterning a $1 \mu \mathrm{m} \mathrm{SiRN} \mathrm{layer} \mathrm{on} \mathrm{top} \mathrm{of} \mathrm{the} \mathrm{poly-silicon.} \mathrm{Aluminium}(80 \mathrm{~nm})$ is sputtered 


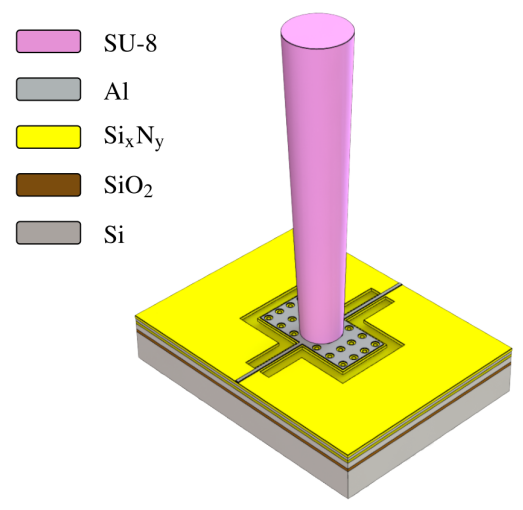

(a)

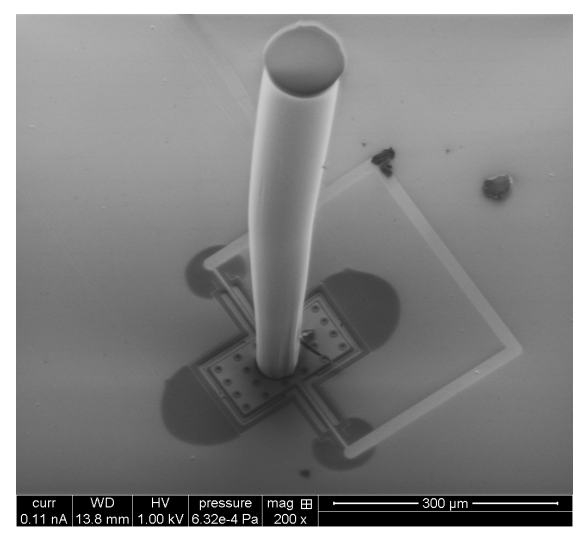

(b)

Figure 4. Design (a) and fabrication (b) of the MEMS hair-based accelerometer fabricated by surface micromachining and using SU-8 lithography.

on top of the membrane to create the electrodes for capacitive read-out. Our artificial clavate hair is created by two layers of SU-8, to realize both the centre of mass towards the top of the hair structure and a total hair length of about $800 \mu \mathrm{m}$ with an average diameter of about $80 \mu \mathrm{m}$. Finally, to release the membrane the sacrificial poly-silicon layer is removed using $\mathrm{XeF}_{2}$ etching. The fabrications results are shown by the SEM image in figure $4 \mathrm{~b}$.

\subsection{Experimental}

3.4.1. Setup Experiments to measure the sensor's response are performed using the setup shown in figure 5. A waveform generator (Agilent 33220A-001) is used to produce a sinusoidal signal at a frequency $f(\omega=2 \pi f)$ that is supplied to an amplifier. This amplifier drives a voice-coil shaker (MB Electronics PM 50) to generate harmonic acceleration. Carrier signals at $1 \mathrm{MHz}$ are supplied to the bottom electrodes of the accelerometer for capacitive read-out. The top electrode is connected to a charge amplifier, and after demodulation and filtering (Stanford SR 650) the sensor's response is monitored on an oscilloscope (Agilent DS1024). For calibration, the applied accelerations were measured by mounting a reference accelerometer (STEVAL-MKI021V1) on the shaker.

3.4.2. Frequency response First, the frequency response of the hair-based accelerometer was measured using capacitive read-out in the direction perpendicular to the rotational axis. Frequencies within a range of $50-1000 \mathrm{~Hz}$ were applied to the shaker. The reference accelerometer was used to determine the externally applied acceleration amplitude. The resulting measured magnitude response of the bio-inspired accelerometer is shown in red in figure 6. Here, the circles represent the measurements and the dashed line exhibits the analytical model based on (1), where the resonance frequency $\omega_{0}$ and the quality factor $Q$ were fitted. We observe good agreement between model and measurements, where the resonance frequency is found to be about $320 \mathrm{~Hz}$. 


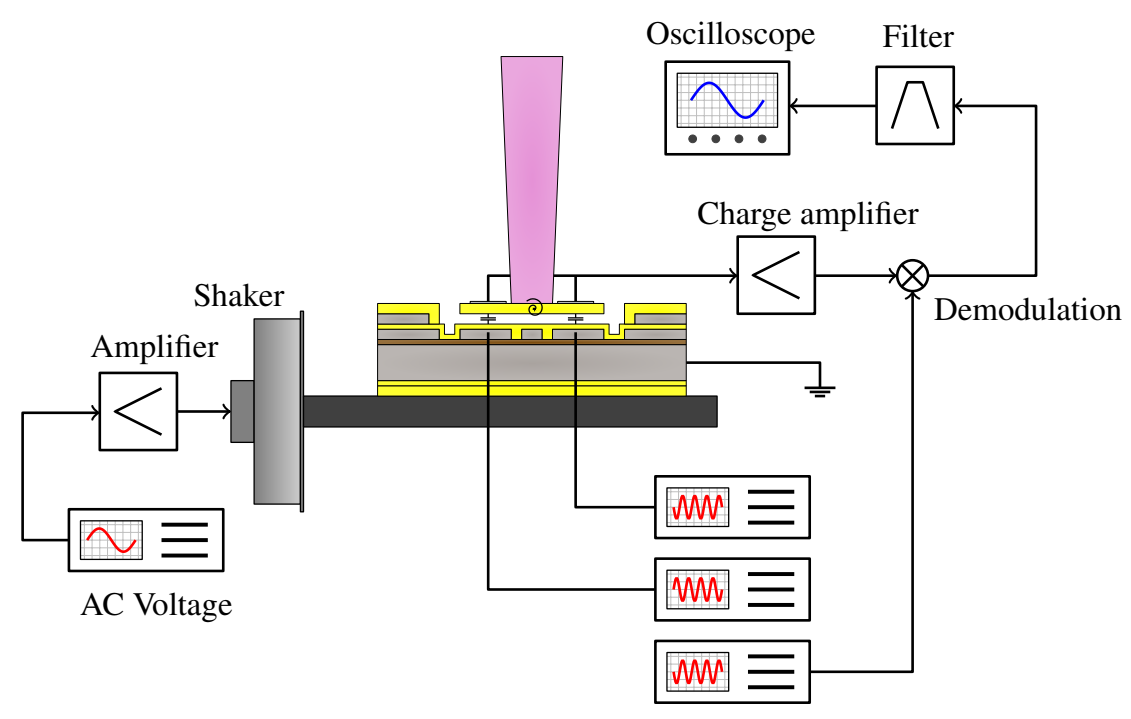

Carrier signals

Figure 5. Capacitive measurement setup for determining the membrane rotation of the biomimetic accelerometer.

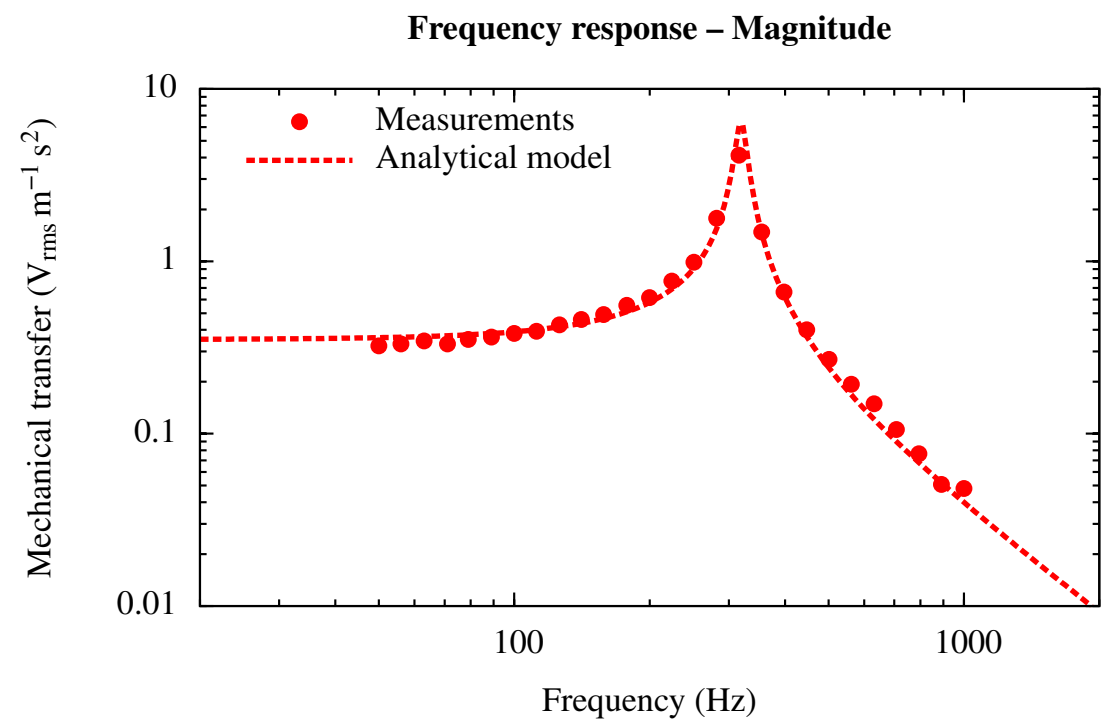

Figure 6. Measured mechanical transfer of the hair-based accelerometer using capacitive read-out.

3.4.3. Directivity The sensor's directivity was measured by rotating it over $360^{\circ}$, with steps of $10^{\circ}$, with respect to the direction of the applied external acceleration, while using capacitive read-out. To this end, a harmonic acceleration with a frequency of $80 \mathrm{~Hz}$ was applied and the output voltage was measured by a multimeter (Keithley 2000). The obtained results are shown in figure 7 . We observe that the measurements are in close agreement with the theoretical response for a so-called figure-of-eight. The measurements indicate that the hair- 


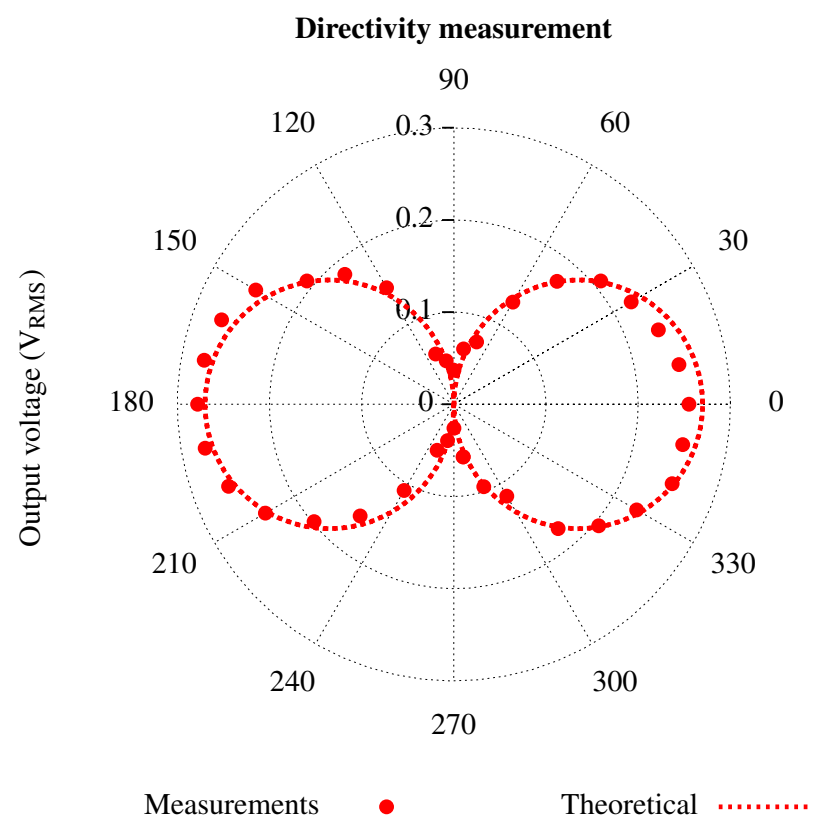

Figure 7. Measured directivity of the hair-based accelerometer using capacitive read-out at an acceleration frequency of $80 \mathrm{~Hz}$.

based accelerometer has a maximum responsivity for both $0^{\circ}$ and $180^{\circ}$, which coincides with the direction perpendicular to the rotational axis of the hair sensor.

3.4.4. Threshold and linearity To obtain a model which describes the sensor's signal-tonoise ratio (SNR) as a function of acceleration amplitude as well as the sensor's detection threshold, the signal and noise powers are considered. The signal is assumed to have a linear relationship with respect to the acceleration amplitude $a_{0}$, given by the coefficient $S_{\mathrm{c}}(\omega)$. This coefficient is directly related to the sensor's rotational angle $\theta(\omega)$ and therefore has a dependency on the acceleration frequency $\omega . N_{\mathrm{c}}$ is a constant representing the total noise, giving for the measured output $z$ due to the uncorrelated sources:

$$
z(\boldsymbol{\omega})=\sqrt{\left(S_{\mathrm{c}}(\boldsymbol{\omega}) a_{\mathrm{ext}}\right)^{2}+N_{\mathrm{c}}^{2}} .
$$

From this expression, the measured detection threshold $a_{\min }$ can be found by equating the noise and signal powers (or alternatively by calculating the intersection value of the asymptotes) which requires only the values for $S_{\mathrm{c}}$ and $N_{\mathrm{c}}$ :

$$
a_{\min }(\omega)=\frac{N_{\mathrm{c}}}{S_{\mathrm{c}}(\omega)} .
$$

Experiments to determine the sensor's linearity were performed by choosing first a specific acceleration frequency $(80 \mathrm{~Hz})$ and then by varying the acceleration amplitude. Subsequently, from the measured output rms-voltage the sensor's detection limit and linearity are derived. The results are shown in figure 8 , where the points represent the measurements, the solid 
Linearity and noise level at $80 \mathrm{~Hz}$

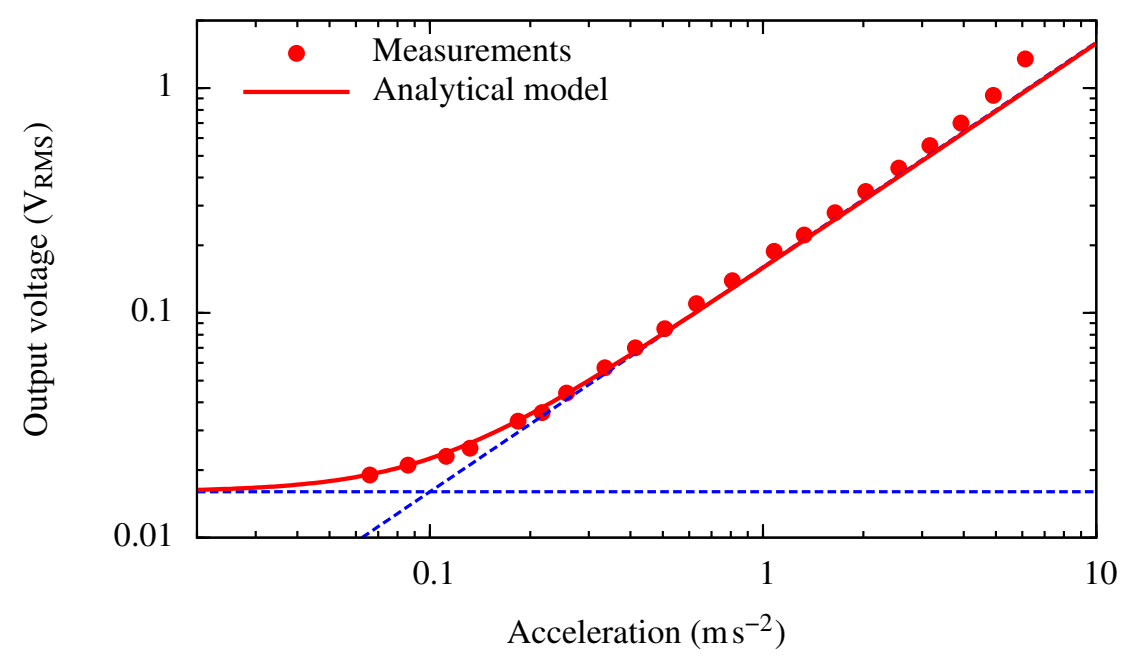

Figure 8. Measured response versus acceleration amplitudes at a frequency of $80 \mathrm{~Hz}$ using capacitive read-out.

line is based on (9), and the dashed lines indicate the constant equivalent noise amplitude and ideal linear response asymptotes. We observe that for accelerations with an amplitude of more than $0.1 \mathrm{~m} \mathrm{~s}^{-2}$, indicated by the intersection of the asymptotes, the hair-based accelerometer exhibits a clear linear relationship with the applied acceleration. Below this amplitude, the sensor's output is dominated by noise $(\mathrm{SNR}<1)$.

To get some insight in the accelerometer's noise performance and stability, an Allan variance measurement was performed. The zero-acceleration output rms-voltage was measured with a time interval of $20 \mathrm{~ms}$ for a period of $2 \mathrm{~h}$ using a multimeter (Agilent 34401A) connected to LabVIEW. The results of the subsequently calculated Allan deviation are shown in figure 9, together with asymptotic lines for both the velocity random walk and the bias instability.

From the linearity measurements, the error on full-scale (i.e. the measurement taken at highest acceleration of $6.12 \mathrm{~m} \mathrm{~s}^{-2}$, see figure 8) was calculated and found to be $3.3 \%$. By considering the detection threshold and the full scale acceleration amplitude, the dynamic range of the hair-based accelerometer is about $35.6 \mathrm{~dB}$. The Allan variance results showed a velocity random walk of $1.67 \mathrm{~m} \mathrm{~s}^{-1} \sqrt{\mathrm{h}}^{-1}$ and a bias instability of $5 \times 10^{-3} \mathrm{~m} \mathrm{~s}^{-2}$. To summarize, an overview of the sensor performance is shown in table 1.

\subsection{Discussion}

The measured threshold of the bio-inspired accelerometer is about $0.10 \mathrm{~m} \mathrm{~s}^{-2}$, which is about a factor 100 larger than the calculated thermal noise based value of $1.1 \times 10^{-3} \mathrm{~m} \mathrm{~s}^{-2}$ (Gabrielson, 1993). The relative large detection threshold is due to the presence of comparatively strong noise sources in the read-out electronics (Dagamseh et al., 2013). Improvement of these 


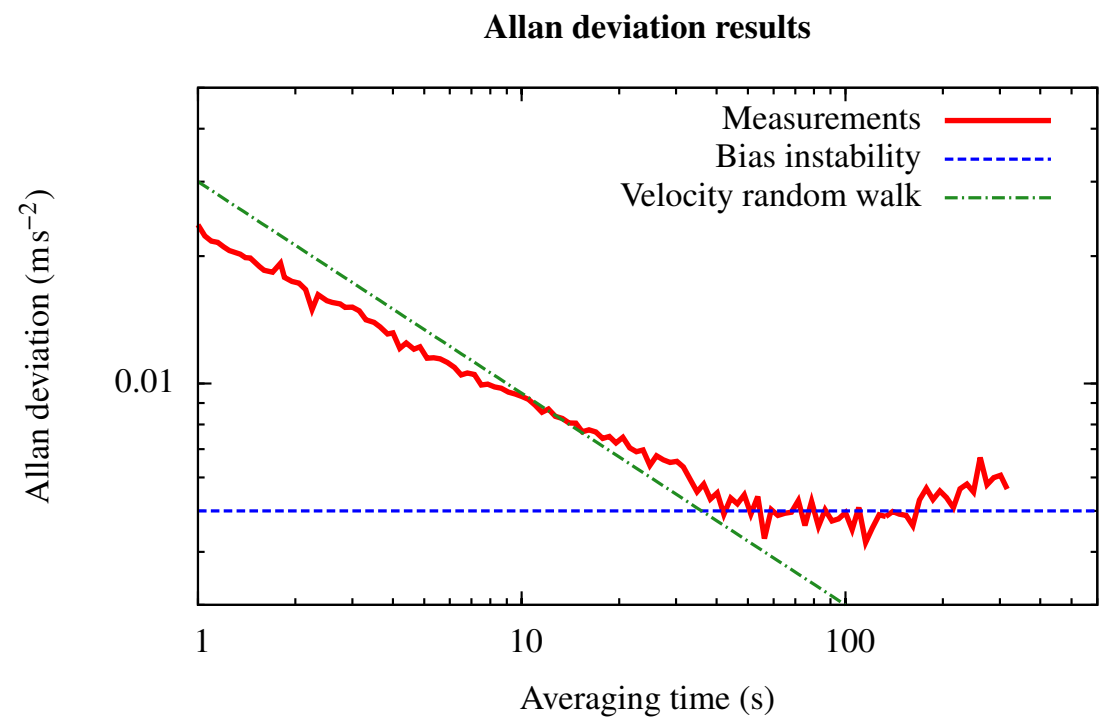

Figure 9. Measured Allan deviation using capacitive read-out.

Table 1. Experimental values of the bio-inspired hair-based accelerometer.

\begin{tabular}{lll}
\hline Quantity & Symbol & Value \\
\hline \multicolumn{2}{l}{ Mechanical parameters } & \\
\hline Quality factor & $Q$ & 12.5 \\
Resonance frequency & $f_{0}$ & $319.6 \mathrm{~Hz}$ \\
\hline Sensor performance $(80 \mathrm{~Hz})$ & \\
\hline Threshold & $a_{\mathrm{min}}$ & $0.10 \mathrm{~m} \mathrm{~s}^{-2}$ \\
Dynamic range & $\mathrm{DR}$ & $\geq 35.6 \mathrm{~dB}$ \\
Full scale error & $\varepsilon_{\mathrm{FS}}$ & $3.3 \%$ \\
Velocity random walk & $\mathrm{VRW}$ & $1.67 \mathrm{~m} \mathrm{~s}^{-1} \sqrt{\mathrm{h}}^{-1}$ \\
Bias instability & $\sigma_{\mathrm{b}}$ & $5 \times 10^{-3} \mathrm{~m} \mathrm{~s}^{-2}$ \\
Thermal noise limit & $a_{\mathrm{th}}$ & $1.1 \times 10^{-3} \mathrm{~m} \mathrm{~s}^{-2}$ \\
\hline
\end{tabular}

electronics, by reduction of its noise, will help to bring the detection threshold for the accelerometer towards the thermal noise limited threshold. Similar differences between measured and (calculated) thermal noise levels have been observed for our previously reported bio-inspired hair flow sensors (Droogendijk et al., 2012b; Dagamseh et al., 2013).

Generally, the susceptibility for (gravitational) acceleration is used by crickets for determination of their position and orientation. The hair-based accelerometer described in this work allows in principle also for determination of orientation using the Earth's gravitational field. That is, by measuring the projection of the Earth's gravitational acceleration, the angle of rotation of the accelerometer with respect to Earth can be determined. However, since the fabricated accelerometer has limits with respect to resolution, an error in this angle will result. Based on the experimental data listed in table 1, this error is calculated to be in the order of 
$0.7^{\circ}$ for accelerations well below resonance $(\omega \rightarrow 0)$, which emphasizes the potential use of this accelerometer to determine orientation. Notice that this value approaches the resolution of the cricket's clavate hair system of $0.1^{\circ}$ (Bischof, 1975).

As we have shown in figure 7, the hair-based accelerometer has a strong directivity. In our MEMS version, this directivity stems from both the mechanical design, which primarily allows rotation around the torsional axis of the sensor, and the differential capacitive read-out, which causes a strong reduction of signals caused by tilting of the hair. As a consequence, multiple hair-based accelerometers may be used simultaneously to sense acceleration in 3D. In crickets, filiform hairs have been shown to have preferential directions of rotations with ratios in stiffness of 'hard' over 'easy' directions between 4 and 8 (Kanou et al., 1989). Bischof (1974) demonstrated that such directivity exists in the cricket's clavate hairs. Additionally, Sakaguchi and Murphey (1983) have shown that crickets use the many clavate hair-sensors on their cerci for determination of their orientation relative to the gravitational field and that they do so both with respect to roll (rotation around longitudinal axis of the animal) and pitch.

\section{Fly-inspired gyroscope}

\subsection{Physics}

The dynamics of the haltere-based gyroscope and common MEMS gyroscopes are similar, since both types consist of vibrating structures governed by two coupled second order differential equations. The design of these gyroscopes is in general a 2-D isotropic, damped mass-spring system. To describe the haltere mechanics, we first define the force $\vec{F}$ acting on the haltere knob (Nalbach, 1993):

$$
\vec{F}=m \vec{g}-m \ddot{\overrightarrow{r_{\mathrm{i}}}}-m \vec{a}_{\mathrm{f}}-m \dot{\vec{\Omega}} \times \vec{r}_{\mathrm{i}}-m \vec{\Omega} \times\left(\vec{\Omega} \times \vec{r}_{\mathrm{i}}\right)-2 m \vec{\Omega} \times \dot{\vec{r}}_{\mathrm{i}},
$$

where $\times$ denotes the vector product, $m$ is the mass of the knob, $\vec{g}$ is the gravitational acceleration, $\vec{r}_{\mathrm{i}}$ is the position of the knob with respect to the fly, $\vec{a}_{\mathrm{f}}$ is the linear acceleration

of the fly in space, and $\vec{\Omega}$ is the angular rate. Six forces contribute to the total inertial force: gravitational $(m \vec{g})$, primary $(m \ddot{\vec{r}})$, linear acceleration $(m \vec{a})$, angular acceleration $\left(m \dot{\vec{\Omega}} \times \vec{r}_{\mathrm{i}}\right)$, centrifugal $\left(m \vec{\Omega} \times\left(\vec{\Omega} \times \vec{r}_{\mathrm{i}}\right)\right)$ and Coriolis $\left(-2 m \vec{\Omega} \times \dot{\vec{r}}_{\mathrm{i}}\right)$.

Now, we assume that linear accelerations are absent $\left(\vec{a}_{\mathrm{f}}=0\right)$, gravitational acceleration $\vec{g}$ is only present in the $z$-direction (the axial direction of the haltere), angular rates are applied only in the horizontal $x y$-plane $\left(\Omega_{x}=\Omega_{y}=0\right)$, the movement of the haltere is harmonic with frequency $\omega$ and the angular rate $\Omega$ is constant with value $\Omega_{0}$.

$$
\Omega=\Omega_{0} .
$$

Consequently, the force amplitude $\vec{F}$ for the $x$ and $y$-direction, since the $z$-direction is not of interest to us, is given by:

$$
\vec{F}_{0}=m\left[\begin{array}{cc}
\omega^{2}+\Omega_{0}^{2} & -j 2 \omega \Omega_{0} \\
j 2 \omega \Omega_{0} & \omega^{2}+\Omega_{0}^{2}
\end{array}\right]\left[\begin{array}{l}
x_{0} \\
y_{0}
\end{array}\right]
$$




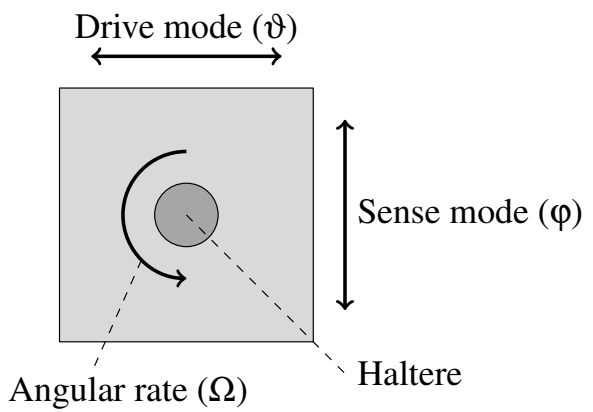

Figure 10. Operation principle of the biomimetic gyroscope (top view).

where $x_{0}$ and $y_{0}$ denote the amplitudes in the $x$ and $y$-direction respectively. Now, the coordinate system is changed into cylindrical coordinates, as shown in figure 10. The system is continuously driven in a steady-state rotational motion in the drive mode with angle $\vartheta$. Rotation around the haltere induces Coriolis-based motion in the sense mode, with the angle $\varphi$ proportional to the input angular rate $\Omega$. By using the following relationships, the force acting on the haltere can be written as a torque $\vec{T}$ for small angle amplitudes $\vartheta_{0}$ and $\varphi_{0}$ :

$$
J=\eta m L^{2}, \quad x_{0}=L \sin \left(\vartheta_{0}\right) \approx L \vartheta_{0}, \quad y_{0}=L \sin \left(\varphi_{0}\right) \approx L \varphi_{0},
$$

where $L$ is the haltere length, $J$ is the moment of inertia and $\eta$ is a parameter depending on the precise geometry. Here, $\eta=1$ by considering the haltere knob as a point mass. As a result, the torque acting on the haltere for constants angular rates becomes

$$
\overrightarrow{\hat{T}}=J\left[\begin{array}{cc}
\omega^{2}+\Omega_{0}^{2} & -j 2 \omega \Omega_{0} \\
j 2 \omega \Omega_{0} & \omega^{2}+\Omega_{0}^{2}
\end{array}\right]\left[\begin{array}{l}
\vartheta_{0} \\
\varphi_{0}
\end{array}\right]
$$

The torque $\vec{T}$ acts on the haltere, which is part of the isotropic gyroscopic mechanical system. The response of this system is described by a coupled second order differential equation:

$$
\left[\begin{array}{cc}
G(\omega) & -C(\omega) \\
C(\omega) & G(\omega)
\end{array}\right]\left[\begin{array}{l}
\vartheta_{0} \\
\varphi_{0}
\end{array}\right]=\left[\begin{array}{c}
\mathcal{T}_{\mathrm{ext}} \\
0
\end{array}\right]
$$

where $G(\omega)$ is given by:

$$
G(\omega)=-\omega^{2}+j 2 \zeta \omega \omega_{0}+\omega_{0}^{2}-\Omega_{0}^{2}
$$

and $C(\omega)$ denotes the coupling between the drive and sense mode due to angular rates:

$$
C(\omega)=j 2 \omega \Omega_{0}
$$


In these expressions, the resonance frequency $\omega_{0}$, damping ratio $\zeta$ and normalized driving torque $\mathcal{T}_{\text {ext }}$ are defined as

$$
\omega_{0}=\sqrt{\frac{S}{J}}, \quad \zeta=\frac{R}{2 \sqrt{S J}}, \quad \mathcal{T}_{\text {ext }}=\frac{T_{0}}{J} .
$$

where $T_{0}$ is the driving torque $\S, S$ is the torsional stiffness, $R$ is the torsional resistance and $J$ is the moment of inertia. Further, $\vartheta_{0}$ and $\varphi_{0}$ are the amplitudes of respectively drive and sense mode.

From (16) follows that the exposure to an angular rate $\Omega$ clearly affects the system's behaviour; its resonance frequency is reduced by centrifugal forces, and torque contributions by angular Coriolis forces cause coupling between drive and sense mode. Although the reduction in resonance frequency by $\Omega_{0}^{2}$ is typically negligible, the principle of a ratedependent resonance frequency can be used for gyroscopic measurement of large angular rates (Zotov et al., 2011).

\subsection{Design}

The governing equation of motion for the drive mode with amplitude has already been stated in (16). By driving the gyroscope by a harmonic torque, assuming that the angular rate $\Omega$ is small compared to the sensor's resonance frequency $\left(\Omega_{0} \ll \omega_{0}\right)$, and angular accelerations are small, the drive mode amplitude $\vartheta_{0}$ can be approximated well by:

$$
\vartheta_{0}(\omega)=\frac{\mathcal{T}_{\text {ext }}}{\sqrt{\left(\omega_{0}^{2}-\omega^{2}\right)^{2}+\left(2 \zeta \omega_{0} \omega\right)^{2}}} .
$$

Generally, MEMS gyroscopes are driven at a frequency $\omega$ equal to the gyroscope's resonance frequency $\omega_{0}$ in the case of underdamped systems. Then, the drive mode amplitude can be simplified to:

$$
\vartheta_{0}=\frac{\mathcal{T}_{\mathrm{ext}}}{2 \zeta \omega_{0}^{2}}
$$

Based on the drive mode motion, the normalized Coriolis-induced torque amplitude $\mathcal{I}_{\mathrm{c}}$ of the sense mode is given by:

$$
\mathcal{T}_{\mathrm{c}}=2 \omega_{0} \vartheta_{0} \Omega_{0} .
$$

Using the system of equations of (16), the corresponding sense mode amplitude $\varphi_{0}$ can be approximated as:

$$
\varphi_{0}=\frac{\mathcal{T}_{\text {ext }}}{2} \frac{1}{\omega_{0}^{3} \zeta^{2}} \Omega_{0} .
$$

$\S$ This torque is delivered by electrostatic actuation in the case of the MEMS gyroscope and by the muscles in case of the fly. 
Now, we define the ratio $\mathcal{R}$ between sense and drive mode amplitudes, which is a measure for the system's responsivity\|. Since Coriolis forces in general are small, a large $\mathcal{R}$ is desirable in order to measure correspondingly small angular rates $\Omega$. The ratio $\mathcal{R}$ is defined as:

$$
\mathcal{R}=\frac{\mathrm{d}}{\mathrm{d} \Omega_{0}}\left(\frac{\varphi_{0}}{\vartheta_{0}}\right)=\kappa
$$

where $\kappa$ is a design parameter, which is the reciprocal of the product of damping ratio $\zeta$ and resonance frequency $\omega_{0}$ :

$$
\kappa=\frac{1}{\zeta \omega_{0}} .
$$

However, in case the gyroscope is critically damped or overdamped, the gyroscope should be operated below its resonance frequency $\omega_{0}$. A suitable frequency of operation is when the damping term of (19) becomes equal to the stiffness-related term for small angular rates $\Omega$, and the actuation frequency is chosen half this frequency:

$$
2 \zeta \omega_{0} \omega=\omega_{0}^{2}, \quad \text { giving } \quad \omega=\frac{\omega_{0}}{2 \zeta} .
$$

By operating the device in such a way, in both the drive and sense mode the factor $2 \zeta$ drops, but the expression for the Coriolis torque given by (22) remains unchanged. Therefore, by this analysis, the ratio of the drive and sense mode amplitudes of a gyroscope is equal for all classes of damping.

The performance of a gyroscope is also to be determined by its response time; besides a sensitive sensory system, also the ability to quickly respond to changes in angular rate $\Omega$ classifies the system. The corresponding response time $\tau_{95}$ - a measure for agility - is calculated from the second order differential equation, describing the mechanical behaviour of the system in response to a torque step function. Here, we assume that the gyroscope is driven in steady state with frequency $\omega$, the coupling from sense to drive mode is negligible, and an angular rate $\Omega$ is applied stepwise. In that case, the governing equation for analyzing transients in the sense mode becomes

$$
\frac{\mathrm{d}^{2} \varphi(t)}{\mathrm{d} t^{2}}+2 \zeta \omega_{0} \frac{\mathrm{d} \varphi(t)}{\mathrm{d} t}+\left(\omega_{0}^{2}-\Omega_{0}^{2}\right) \varphi(t)=2 \omega \Omega_{0} \vartheta_{0} \sin (\omega t) H(t),
$$

where $H(t)$ denotes the Heaviside step function. The general solution of this damped second order system is:

$$
\varphi(t)=\Phi_{1} e^{-r_{1} t}+\Phi_{2} e^{-r_{2} t}
$$

where $\Phi_{1}$ and $\Phi_{2}$ need to be derived from initial conditions and $r_{1}$ and $r_{2}$ are given by:

$$
r_{1,2}=\zeta \omega_{0} \pm \sqrt{\zeta^{2} \omega_{0}^{2}-\omega_{0}^{2}+\Omega_{0}^{2}}
$$

|| This ratio is related to the scale factor of MEMS gyroscopes by taking the transfer by capacitive read-out into account (IEEE Aerosp. Electron. Sys. Soc., 2001). 
which simplifies under the condition $\Omega_{0} \ll \omega_{0}$ to

$$
r_{1,2} \approx \omega_{0}\left(\zeta \pm \sqrt{\zeta^{2}-1}\right) .
$$

The general solution (28) is either real or complex. When the damping ratio $\zeta$ is equal or greater than one, the solution is real and the hair moves without oscillations towards the point of torque equilibrium, dominated by the slow moving mathematical term. When the damping ratio $\zeta$ becomes smaller than one, the square root term becomes imaginary and the hair shows overshoot towards its equilibrium, leading to a longer stabilization time. The response time $\tau_{95}$, indicated as the time to get stable within $95 \%$ of the end value, is approximatedgl as:

$$
\tau_{95} \approx \begin{cases}\frac{3}{\zeta \omega_{0}} & \text { if } \zeta<0.5, \\ \frac{6 \zeta}{\omega_{0}} & \text { if } \zeta>1 .\end{cases}
$$

The best response time is obtained when $\zeta=0.707$, corresponding to a close to critically damped system (Shinners, 1998). However, we observe that nearly all described gyroscopic systems shown in figure 11 are clearly underdamped.

The sense/drive ratio and response time are taken as a criterion for designing a gyroscope. However, these quantities exhibit a similar proportionality with respect to the design parameter $\kappa$ for underdamped gyroscopes:

$$
\tau_{95} \propto \kappa, \quad \mathcal{R} \propto \kappa,
$$

As a consequence, a trade-off exists between the sensitivity parameter $R$ and the response time $\tau_{95}$ (see also (Apostolyuk et al., 2002)). Namely, to have a good sensitivity $\mathcal{R}$, a large $\kappa$-value is required, but to obtain a fast responding system, $\kappa$ should be small. For design and analysis, we capture this trade-off in a single number by defining a figure of merit:

$$
\text { Figure of merit }=\frac{\text { Sense/drive ratio }}{\text { Response time }}=\frac{\mathcal{R}}{\tau_{95}} .
$$

An overview of several MEMS gyroscopic systems and the blowfly's haltere-based gyroscope (Nalbach, 1993) is shown in figure 11 by evaluating the figure of merit as function of the damping ratio $\zeta$. We observe that most MEMS gyroscopes are clearly designed with focus on achieving high responsivity at the cost of bandwidth. Especially the gyroscopes described in (Trusov et al., 2011; Prikhodko et al., 2011) show very low damping factors while having a resonance frequency at $2 \mathrm{kHz}$, being highly sensitive to angular rate, since the ultimate purpose of these two gyroscopes is angle measurement rather than angular rate measurement. Contrarily, the fly's haltere is clearly geared towards a fast response. Also, MEMS-based gyroscopes typically have operating frequencies which are $1 \mathrm{kHz}$ or more, which is significantly higher than the $150 \mathrm{~Hz}$ of the blowfly (Nalbach, 1993). Notice that II A precise mathematical solution is rather involved. A spline interpolation is used for connecting the two asymptotes smoothly and obtaining a good approximation, which is not shown here. 


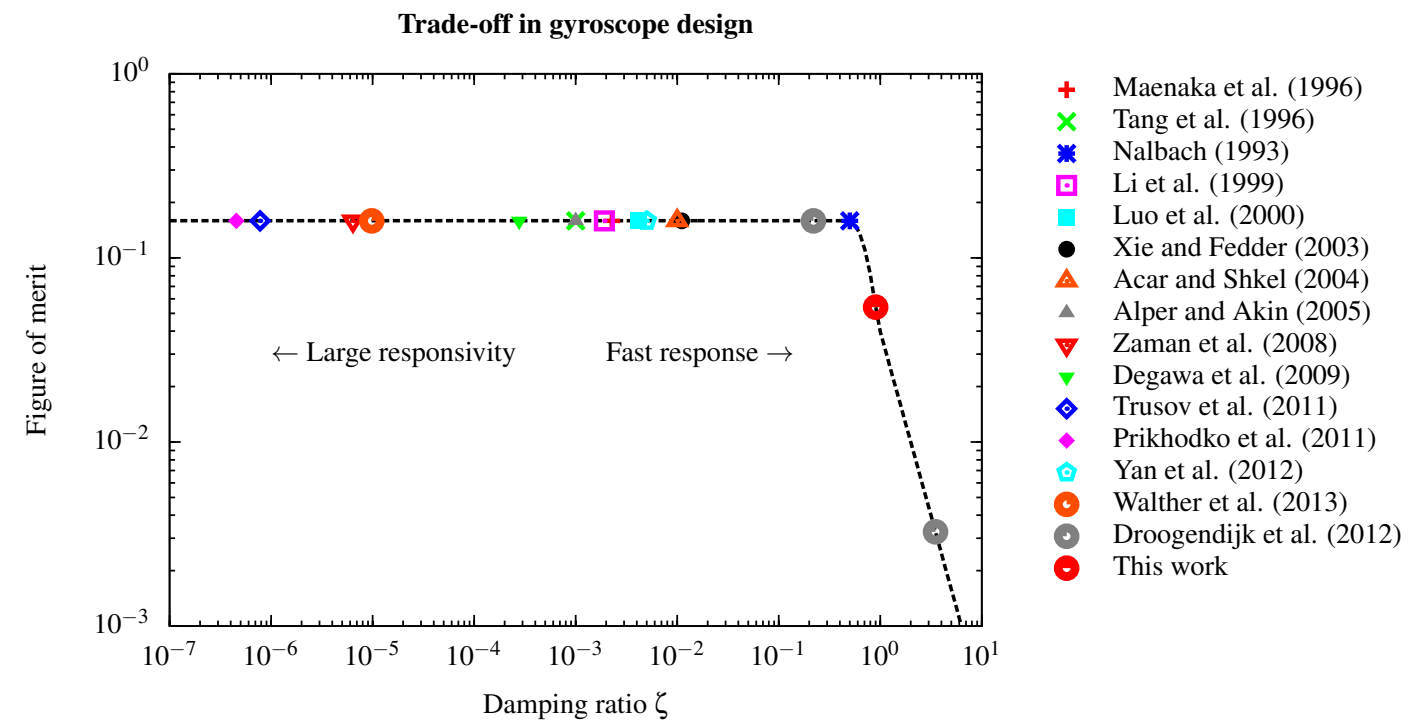

Figure 11. Figure of merit for designing a gyroscopic system. The dashed line indicates the figure of merit by (33).

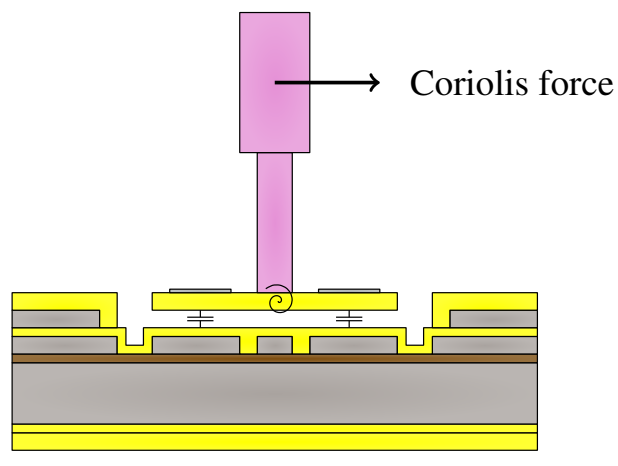

Figure 12. Schematic view of the biomimetic gyroscope (side view).

the figure of merit exhibits a clear decrease when having an overdamped system $(\zeta>1)$, implying that vibratory gyroscopes should preferably be underdamped $(\zeta<1)$.

The eventual design of the bio-inspired gyroscope is based upon the fabrication process for bio-inspired hair flow sensors described by Bruinink et al. (2009) and Dagamseh et al. (2011) to realize a haltere-like structure. Our gyroscope will be actuated in the drive mode with angle $\vartheta$ (see figure 10) by exploiting electrostatic actuation. Electrostatic forces induced by judiciously chosen ac-voltages bring the artificial haltere in a steady-state resonant rotational motion. Measurement of the Coriolis-induced movement of the artificial haltere in the sense direction with rotational angle $\varphi$ is achieved by measuring the differential change in capacitance upon tilt of the artificial haltere (figure 12).

Although we reported on the design and fabrication of such a gyroscope earlier (Droogendijk et al., 2012a), we implemented some important design adjustments here. The suspension of the haltere-based gyroscope is changed to a gimbal-suspension, like the 


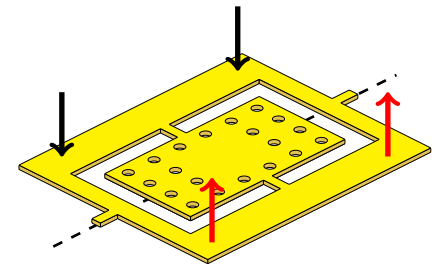

(a) Drive mode.

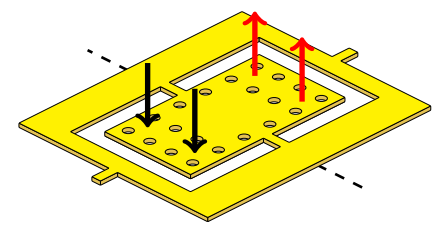

(b) Sense mode.

Figure 13. Characteristic modes of the gimbal gyroscope.

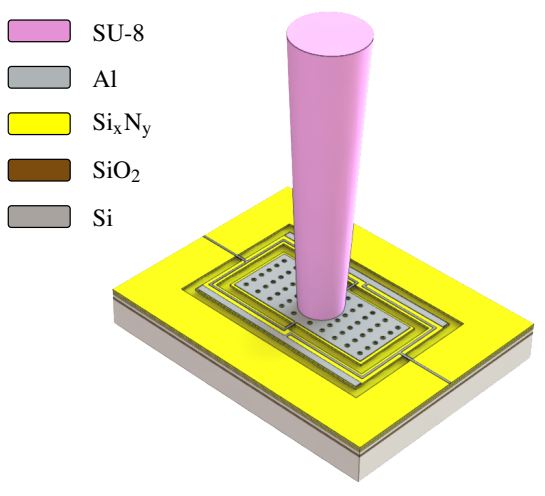

(a)

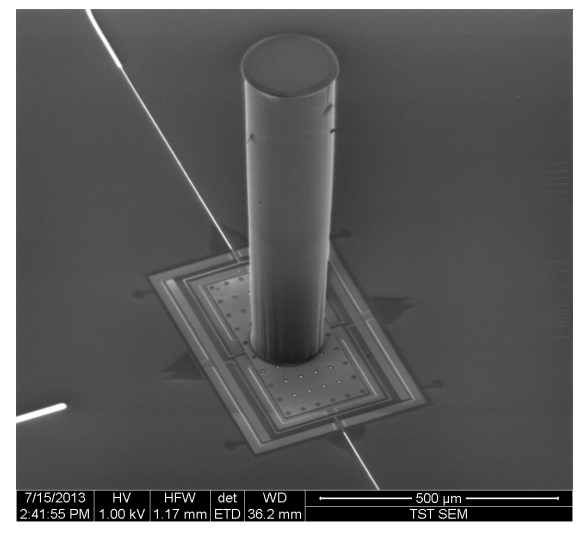

(b)

Figure 14. Design (a) and fabrication (b) of the MEMS haltere-based gyroscope fabricated by surface micromachining and using SU-8 lithography.

gyroscope described by Acar and Shkel (2004). Doing so, the effects of residual stress by having a bi-layer suspension (i.e. $\mathrm{SiRN}$ and $\mathrm{Al}$ ) can be significantly reduced. Also, the drive and sense modes get mechanically distinguishable, allowing for a separate optimisation of both modes. A schematic view of the two modes for our gimbal suspension is given in figure 13. A disadvantage of such a gimbal-design is the difficulty to achieve mode-matching (between drive and sense), which is often aimed for in MEMS gyroscopes to achieve better performance (see e.g. (Hu et al., 2011; Sonmezoglu et al., 2012)). However, on following the bio-inspired approach, the quality factor $Q$ of a gyroscope is relatively low, and consequently mode-matching plays a less pivotal role for the gyroscope's performance.

\subsection{Fabrication}

An overview of the design and the fabrication process for the bio-inspired gyroscope is shown in figure 14a. The fabrication process is identical to the one shown for the bio-inspired accelerometer described in section 3.3. The fabrication results for the gyroscope are shown by the SEM image in figure $14 \mathrm{~b}$. 


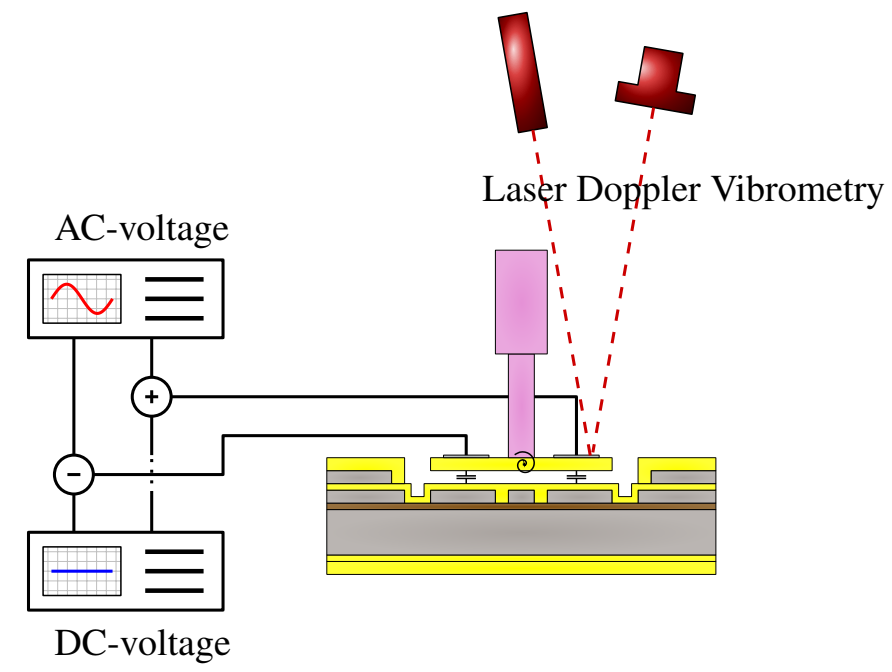

Figure 15. Experimental setup for characterization of the biomimetic gyroscope.

\subsection{Experimental}

4.4.1. Setup To characterize the mechanical properties of the gyroscope, an experimental setup based on Laser Doppler Vibrometry (Polytec, 2005) is used (figure 15). To measure the rotational modes we differentially applied a sinusoidal signal superposed to a dc-offset voltage (Delta Elektronika - Power Supply E 030-1) to a pair of electrodes.

4.4.2. Frequency response The response for the drive mode (figure 13a) was measured by applying a chirp-voltage in push-pull configuration. The magnitude and phase response are shown in figure 16, both together with the results of an analytical model based on a classical second-order mechanical system. From both figures, the drive mode turns out to be slightly overdamped $(\zeta=0.9)$ with its resonance frequency found at approximately $550 \mathrm{~Hz}$. Due to the overdamped nature of the system, the bandwidth of the drive mode is calculated by computing the intersection of the stiffness and damping contributing terms in (16) and is found to be $305 \mathrm{~Hz}$. Further, we observe that there is a good agreement between the analytical model and the measured response for frequencies up to about $800 \mathrm{~Hz}$.

\subsection{Discussion}

Regarding the fabrication process, the SEM image from the bio-inspired gyroscope (figure 14b) shows that we have successfully fabricated artificial haltere-like structure with increased mass at the top. Optical measurements indicate that the haltere-like structure length is about $800 \mu \mathrm{m}$, and that the haltere-like structures have a slight negative tapering towards the membrane. This tapering results from the light intensity profile during exposure of the SU-8 as well as the two step lithography process incorporated in the design. This effectively causes the centre of mass to be located in the upper part of the haltere-like structures, similar to the fly's haltere (figure 2). 

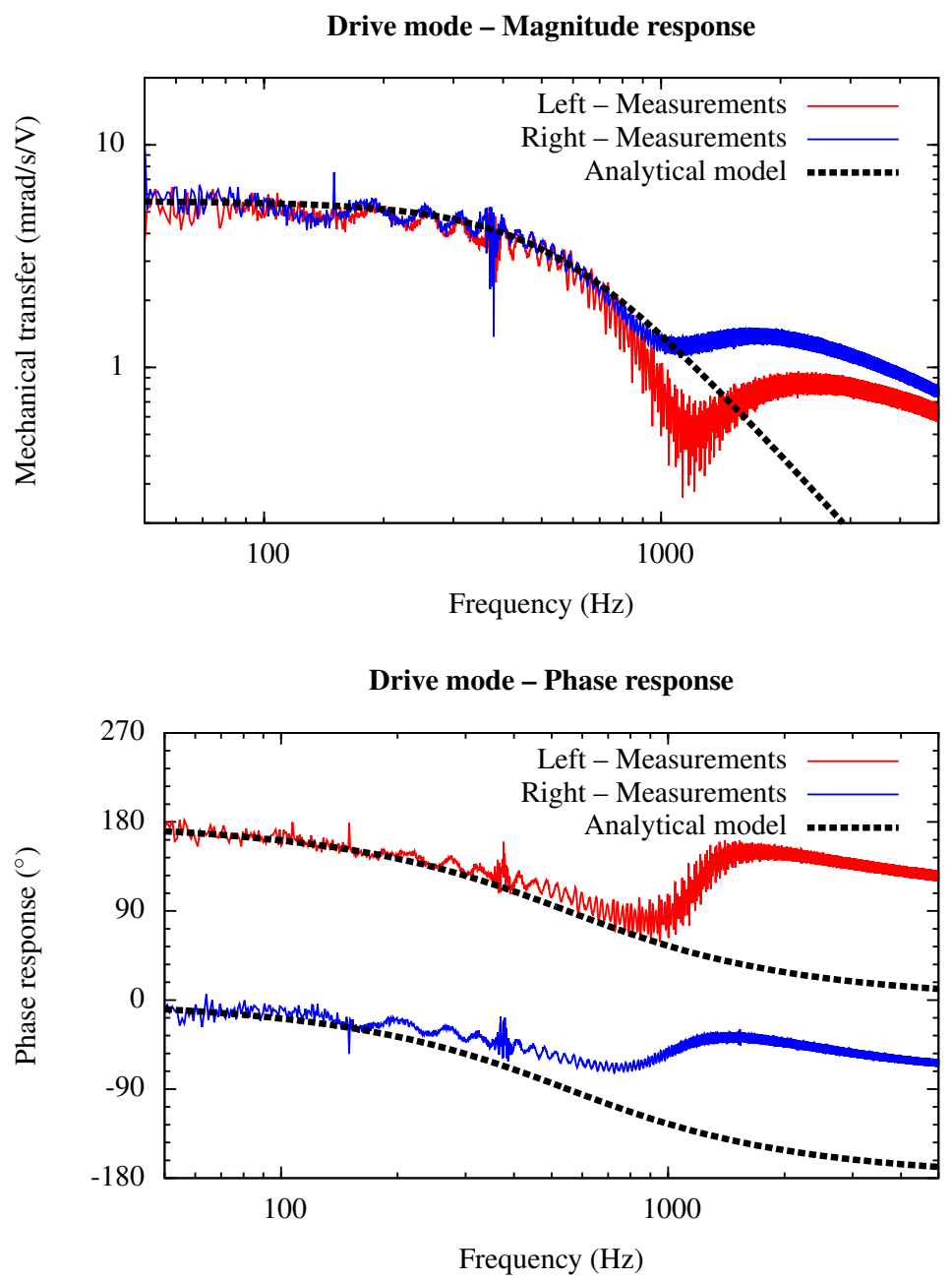

Figure 16. Optically measured response of the membrane displacement by electrostatically actuating the drive mode $\left(f_{0}=550 \mathrm{~Hz}\right.$ and $\left.\zeta=0.9\right)$.

The measured resonance frequency for the drive mode was measured to be about $510 \mathrm{~Hz}$ with a damping ratio of $\zeta=0.9$. Although this resonance frequency is slightly higher than the wing-beat frequency of the fly $(130-150 \mathrm{~Hz})$, it remains in the same order of magnitude. Similar results are obtained for the sense mode, wherein the resonance frequency was found to be about $300 \mathrm{~Hz}$ with a slightly overdamped system $(\zeta=1.1)$. However, we observed that the measured response is a combination of modes, especially for higher excitation frequencies. Since every mode is expected to be about critically damped, it is difficult to identify each modal resonance frequency due to the absence of clear resonance peaks and phase shifts. Theoretically, using capacitive differential read-out techniques, the contributions of the drive and vertical mode can be commonly rejected for measurement of the sense mode.

A haltere-inspired gyroscope has been designed, fabricated and partially characterized. However, we were not able to experimentally demonstrate its response to externally applied angular rates using our home-built rate-table, electrostatic actuation of the drive mode, and 
capacitive read-out of the sense mode. With the current design and the maximum applicable angular rate using our rate table (rate amplitude of about $3000^{\circ} \mathrm{s}^{-1}$ at a frequency of $14 \mathrm{~Hz}$ ), the calculated displacement of the membrane edges for capacitive read-out of the sense mode is in the order of $\mathrm{nm}$, which corresponds to the measurement threshold of our read-out electronics (Dagamseh et al., 2013).

\section{Bio-inspiration continued}

Although a working bio-inspired accelerometer is clearly demonstrated, we believe that a working bio-inspired gyroscope following the 'design rules' of a fly and the use of MEMS technology is difficult to achieve, but also possible. However, this will require adaptation of the design and the fabrication process. For our gyroscope, in particular the value of $\mathcal{R}$ needs to be increased in order to measure the applied angular rates. A possibility is the use of piezoresistive sensing instead of capacitive read-out, as proposed by Wicaksono et al. (2007). Also, improvement of the mechanical gimbal-suspension and the use of more compliant materials will help to increase $\mathcal{R}$. One could also think of exchanging the technology of MEMS for another type of technology (e.g. 3D-printing) and investigate its potential for bio-inspired gyroscopes.

The design and fabrication of two hair-based bio-inspired inertial sensors are described in this chapter: an accelerometer to measure external acceleration and a gyroscope to measure angular rates. A next step in the bio-inspired approach is the combination of hair-based sensors to arrive at a multi-sensor system in which sensor fusion can take place, by adding e.g. the capability for flow sensing (Bruinink et al., 2009). Also, other types of bio-inspired hairbased sensors offer a potential for integration within such hair-based multi-sensor systems, like IR-light sensing (Schmitz et al., 2012) and tactile sensing (Murphey, 1985).

Over the past years, much research has been done on bio-inspired sensors. Despite all effort and the many important scientific results on hair-based sensing, it remains a challenge to engineers and scientists to realize the artificial counterpart of the biological sensor system with equal or better performance. Currently, e.g. insects performs still much better on several aspects (e.g. responsivity and detection threshold), provoking the question as to how to 'beat nature'. To this end, look for improvement of the mechanical design, investigate the use of more compliant materials and consider the (partial) use of different or new technology.

\section{Conclusions}

In biology, hair-based sensor systems are regularly used for measurement of physical quantities. In this chapter, two types of bio-inspired inertial sensors have been discussed: a cricket-inspired accelerometer and a fly-inspired gyroscope. Both the accelerometer and gyroscope have been developed and fabricated using surface micromachining and SU-8 lithography. We showed that this MEMS hair-based accelerometer has a resonance frequency of $320 \mathrm{~Hz}$, a detection threshold of $0.10 \mathrm{~m} \mathrm{~s}^{-2}$ and a dynamic range of more than $35 \mathrm{~dB}$. Further, the accelerometer has clear directivity and a bias instability of $5 \times 10^{-3} \mathrm{~m} \mathrm{~s}^{-2}$. For 
the gyroscope, design rules were investigated and it was shown that haltere-based gyroscopes tend to emphasize a fast response, rather than a high responsivity or low detection threshold, by having a relatively low quality factor. Measurements on our haltere-inspired sensors indicate an excitable gyroscope with a (drive mode) resonance frequency of about $550 \mathrm{~Hz}$ and a damping ratio of 0.9 . A response to externally applied angular rates was not demonstrated due to the limited applied rotation rates and the very small induced sense mode amplitude, which is mainly caused by the structures being overdamped and the small achievable drive mode amplitudes.

\section{Acknowledgements}

This work is carried out within the BioEARS-project, funded by STW/NWO.

\section{References}

C. Acar and A. M. Shkel. Structural design and experimental characterization of torsional micromachined gyroscopes with non-resonant drive mode. J. Micromech. Microeng., 14: 15-25, 2004. doi: 10.1088/0960-1317/14/1/303.

S. E. Alper and T. Akin. A single-crystal silicon symmetrical and decoupled MEMS gyroscope on an insulating substrate. J. Microelectromech. S., 14(4):707-717, Aug. 2005. doi: 10.1109/JMEMS.2005.845400.

V. A. Apostolyuk, V. J. Logeeswaran, and F. E. H. Tay. Efficient design of micromechanical gyroscopes. J. Micromech. Microeng., 12:948-954, 2002. doi: 10.1088/0960-1317/12/6/ 327.

H.-J. Bischof. Verteilung und bewegungsweise der keulenformigen sensillen von gryllus bimaculatus. Biol. Zbl., 93:449-457, 1974.

H.-J. Bischof. Die keulenförmigen sensillen auf den cerci der grille Gryllus bimaculatus als schwererezeptoren. J. Comp. Physiol. A, 98:277-288, 1975. doi: 10.1007/BF00656974.

C. M. Bruinink, R. K. Jaganatharaja, M. J. de Boer, J. W. Berenschot, M. L. Kolster, T. S. J. Lammerink, R. J. Wiegerink, and G. J. M. Krijnen. Advancements in technology and design of biomimetic flow-sensor arrays. In Proc. MEMS 2009, pages 152-155, 2009. doi: 10.1109/MEMSYS.2009.4805341.

A. D. Challoner, R. C. Gutierrez, and T. K. Tang. Cloverleaf microgyroscope with electrostatic alignment and tuning. Patent, 2007. US 7159441.

W. P. Chan, F. Prete, and M. H. Dickinson. Visual input to the efferent control system of a fly's "gyroscope". Science, 280:289-292, Apr. 1998. doi: 10.1126/science.280.5361.289.

N. Chen, C. Tucker, J. Engel, Y. Yang, S. Pandya, and C. Liu. Design and characterization of artificial haircell sensor for flow sensing with ultrahigh velocity and angular sensitivity. $J$. Microelectromech. S., 16:999-1014, 2007. doi: 10.1109/JMEMS.2007.902436.

A. Dagamseh, T. Lammerink, R. Sanders, R. Wiegerink, and G. Krijnen. Towards highresolution flow cameras made of artificial hair flow-sensors for flow pattern recognition. 
In Proc. MEMS 2011, pages 648-651, Cancun, Mexico, Jan. 23-27 2011. doi: 10.1109/ MEMSYS.2011.5734508.

A. M. K. Dagamseh, C. M. Bruinink, R. J. Wiegerink, T. S. J. Lammerink, H. Droogendijk, and G. J. M. Krijnen. Interfacing of differential-capacitive biomimetic hair flow-sensors for optimal sensitivity. J. Micromech. Microeng., 23:035010, 2013. doi: 10.1088/0960-1317/ 23/3/035010.

H. Droogendijk, R. A. Brookhuis, M. J. de Boer, R. G. P. Sanders, and G. J. M. Krijnen. Design and fabrication of a biomimetic gyroscope inspired by the fly's haltere. In Proc. IEEE Sensors 2012, pages 1400-1403, Taipei, Taiwan, Oct. 28-31 2012a. doi: 10.1109/ICSENS.2012.6411134.

H. Droogendijk, C. M. Bruinink, R. G. P. Sanders, A. M. K. Dagamseh, R. J. Wiegerink, and G. J. M. Krijnen. Improving the performance of biomimetic hair-flow sensors by electrostatic spring softening. J. Micromech. Microeng., 22(6):065026, Jun. 2012b. doi: 10.1088/0960-1317/22/6/065026.

H. Droogendijk, R. A. Brookhuis, M. J. de Boer, R. G. P. Sanders, and G. J. M. Krijnen. Towards a biomimetic gyroscope inspired by the fly's haltere using MEMS technology. $J$. R. Soc. Interface, 2014a.

H. Droogendijk, M. J. de Boer, R. G. P. Sanders, and G. J. M. Krijnen. A biomimetic accelerometer inspired by the cricket's clavate hair. J. R. Soc. Interface, 11:20140438, 2014b. doi: 10.1098/rsif.2014.0438.

G. Fraenkel and J. W. S. Pringle. Biological sciences: halteres of flies as gyroscopic organs of equilibrium. Nature, 141:919-920, 1938. doi: 10.1038/141919a0.

T. B. Gabrielson. Mechanical thermal noise in micromachined acoustic and vibration sensors. IEEE Trans. Electron. Devices, 40:903-909, 1993. doi: 10.1109/16.210197.

M. Ginsberg, J. Schiano, M. Kramer, and M. Alleyne. A case study in bio-inspired engineering design: defense applications of exoskeletal sensors. Defense \& Security Analysis, 29(2):156-169, 2013. doi: 10.1080/14751798.2013.787798.

R. Hengstenberg. Controlling the fly's gyroscopes. Nature, 392:757-758, Apr. 1998. doi: $10.1038 / 33796$.

E. Horn and H.-J. Bischof. Gravity reception in crickets: The influence of cereal and antennal afferences on the head position. J. Comp. Physiol. A, 150:93-98, 1983. doi: 10.1007/BF00605292.

Z. X. Hu, B. Gallacher, J. S. Burdess, C. P. Fell, and K. Townsend. Precision mode matching of MEMS gyroscopes by feedback control. In Proc. IEEE Sensors 2011, pages 16-19, Limerick, Ireland, Oct. 28-31 2011. doi: 10.1109/ICSENS.2011.6126998.

J. Humphrey, R. Devarakonda, I. Iglesias, and F. Barth. Dynamics of arthropod filiform hairs. i. Mathematical modeling of the hair and air motions. Phil. Trans.: Bio. Sc., 340:423-444, 1993. doi: 10.1098/rstb.1993.0083.

IEEE Aerosp. Electron. Sys. Soc. IEEE standard for inertial sensor terminology, 2001. IEEE Std. 528-2001. 
M. Kanou, T. Osawa, and T. Shimozawa. Mechanical polarization in the air-current sensory hair of a cricket. Experientia, 45:1082-1083, 1989. doi: 10.1007/BF01950163.

G. Krijnen, A. Floris, M. Dijkstra, T. Lammerink, and R. Wiegerink. Biomimetic micromechanical adaptive flow-sensor arrays. Proc. SPIE, 6592:65920F, 2007. doi: 10.1117/12.721807.

C. Liu. Micromachined biomimetic artificial haircell sensors. Bioinsp. Biomim., 2:162-169, 2007. doi: 10.1088/1748-3182/2/4/S05.

R. K. Murphey. The structure and development of a somatotopic map in crickets: The cereal afferent projection. Dev. Biol., 88:236-246, 1981. doi: 10.1016/0012-1606(81)90167-6.

R. K. Murphey. A second cricket cercal sensory system: bristle hairs and the interneurons they activate. J. Comp. Physiol. A, 156:357-367, 1985. doi: 10.1007/BF00610728.

G. Nalbach. The halteres of the blowfly Calliphora - i. Kinematics and dynamics. J. Comp. Physiol. A, 173:293-300, 1993. doi: 10.1007/BF00212693.

G. Nalbach and R. Hengstenberg. The halteres of the blowfly Calliphora - ii. Threedimensional organization of compensatory reactions to real and simulated rotations. $J$. Comp. Physiol. A, 175:695-708, 1994. doi: 10.1007/BF00191842.

Y. Ozaki, T. Ohyama, T. Yasuda, and I. Shimoyama. An air flow sensor modeled on wind receptor hairs of insects. In Proc. MEMS 2000, pages 531-536, Miyazaki, Japan, 2000. doi: 10.1109/MEMSYS.2000.838573.

Polytec. MSA-400 Micro System Analyzer. Polytec, Waldbronn, Germany, 2005.

I. P. Prikhodko, S. A. Zotov, A. A. Trusov, and A. M. Shkel. Sub-degree-per-hour silicon MEMS rate sensor with 1 million Q-factor. In Proc. Transducers'11, pages 2809-2812, Beijing, China, Jun. 5-9 2011. doi: 10.1109/TRANSDUCERS.2011.5969216.

J. W. S. Pringle. The gyroscopic mechanism of the halteres of Diptera. Philos. Trans. R. Soc. London B., 233(602):347-384, Nov. 1948. doi: 10.1098/rstb.1948.0007.

L. Ristroph, A. J. Bergou, G. Ristroph, K. Coumes, G. J. Berman, J. Guckenheimer, Z. J. Wang, and I. Cohen. Discovering the flight autostabilizer of fruit flies by inducing aerial stumbles. PNAS, 107(11):4820-4824, Mar. 2010. doi: 10.1073/pnas.1000615107.

R. Rosner, M. Egelhaaf, J. Grewe, and A. K. Warzecha. Variability of blowfly head optomotor responses. J. Exp. Biol., 212:1170-1184, 2009. doi: 10.1242/jeb.027060.

M. Sadeghi, R. Peterson, and K. Najafi. Micro-hydraulic structure for high performance biomimetic air flow sensor arrays. In Tech. Digest - IEDM, 6131638, pages 29.4.1-29.4.4, 2011. doi: 10.1109/IEDM.2011.6131638.

D. S. Sakaguchi and R. K. Murphey. The equilibrium detecting system of the cricket: physiology and morphology of an identified interneuron. J. Comp. Physiol. A, 150:141152, 1983. doi: 10.1007/BF00606364.

D. C. Sandeman and H. Markl. Head movements in flies (Calliphora) produced by deflexion of the halteres. J. Exp. Biol., 85:43-60, 1980. 
H. Schmitz, H. Soltner, and H. Bousack. Biomimetic infrared sensors based on photomechanic infrared receptors in pyrophilous ("fire-loving") insects. IEEE Sensors J., 12 (2):281-288, Feb. 2012. doi: 10.1109/JSEN.2010.2076324.

T. Shimozawa and M. Kanou. Varieties of filiform hairs: range fractionation by sensory afferents and cercal interneurons of a cricket. J. Comp. Physiol. A, 155:485-493, 1984. doi: 10.1007/BF00611913.

T. Shimozawa, T. Kumagai, and Y. Baba. Structural scaling and functional design of the cercal wind-receptor hairs of cricket. J. Comp. Physiol. A, 183:171-186, 1998. doi: $10.1007 / \mathrm{s} 003590050245$.

T. Shimozawa, J. Murakami, and T. Kumagai. Cricket wind receptors: thermal noise for the highest sensitivity known. In Sensors and sensing in biology and engineering, pages 145-159. Springer, 2003. doi: 10.1007/978-3-7091-6025-1_10.

S. M. Shinners. Modern control system theory and design. John Wiley \& Sons, Inc., $2^{\text {nd }}$ edition, 1998.

G. L. Smith, S. S. Bedair, B. E. Schuster, W. D. Nothwang, J. S. Pulskamp, C. D. Meyer, and R. G. Polcawich. Biologically-inspired, haltere, angular-rate sensors for micro-autonomous systems. In Proc. SPIE, volume 8373, page 83731K, 2012. doi: 10.1117/12.918678.

S. Sonmezoglu, S. E. Alper, and T. Akin. An automatically mode-matched MEMS gyroscope with $50 \mathrm{~Hz}$ bandwidth. In Proc. MEMS 2012, pages 523-526, Paris, France, Jan. 29 - Feb. 2 2012. doi: 10.1109/MEMSYS.2012.6170231.

T. K. Tang, R. C. Gutierrez, C. B. Stell, V. Vorperian, G. A. Arakaki, J. T. Rice, W. J. Li, I. Chakraborty, K. Shcheglov, and J. Z. Wilcox. A packaged silicon MEMS vibratory gyroscope for microspacecraft. In Proc. MEMS 1997, pages 500-505, Nagoya, Japan, Jan. 26-30 1997. doi: 10.1109/MEMSYS.1997.581911.

Y. Tang, R. L. Peterson, and K. Najafi. Technology for fabricating dense 3-D microstructure arrays for biomimetic hair-like sensors. In Proc. MEMS 2013, pages 355-358, Taipei, Taiwan, Jan. 20-24 2013. doi: 10.1109/MEMSYS.2013.6474251.

J. Tao and X. Yu. Hair flow sensors: from bio-inspiration to bio-mimicking-a review. Smart Mater. Struct., 21:113001, 2012. doi: 10.1088/0964-1726/21/11/113001.

M. Tobias and R. K. Murphey. The response of cercal receptors and identified interneurons in the cricket (Acheta domesticus) to airstreams. J. Comp. Physiol. A, 129:51-59, 1979. doi: 10.1007/BF00679911.

A. A. Trusov, I. P. Prikhodko, S. A. Zotov, and A. M. Shkel. Low-dissipation silicon tuning fork gyroscopes for rate and whole angle measurements. IEEE Sens. J., 11(11):2763-2770, 2011. doi: 10.1109/JSEN.2011.2160338.

Y.-H. Wang, C.-Y. Lee, and C.-M. Chiang. A MEMS-based air flow sensor with a freestanding microcantilever structure. Sensors, 7:2389-2401, 2007. doi: 10.3390/s7102389.

D. H. B. Wicaksono, Y. Chen, and P. J. French. Design and modelling of a bio-inspired MEMS gyroscope. In Proc. ICEEI, pages A-05, Bandung, Indonesia, Jun. 17-19 2007. 
W.-C. Wu and R. Wood. Angular rate sensor using electromechanical haltere. Patent, 2006. US 7107842.

W.-C. Wu, R. J. Wood, and R. S. Fearing. Halteres for the micromechanical flying insect. In Proc. ICRA 2002, pages 60-65, Washington, DC, United States, May 2002. doi: 10.1109/ROBOT.2002.1013339.

H. Xie and G. K. Fedder. Fabrication, characterization, and analysis of a drie CMOS-MEMS gyroscope. IEEE Sens. J., 3(5):622-631, Oct. 2003. doi: 10.1109/JSEN.2003.817901.

N. Yazdi, F. Ayazi, and K. Najafi. Micromachined inertial sensors. Proc. IEEE, 86(8):16401659, 1998. doi: 10.1109/5.704269.

Z.-G. Zhou and Z.-W. Liu. Biomimetic cilia based on MEMS technology. J. Bion. Eng., 5: 358-365, 2008. doi: 10.1016/S1672-6529(08)60181-X.

S. A. Zotov, A. A. Trusov, and A. M. Shkel. Demonstration of a wide dynamic range angular rate sensor based on frequency modulation. In Proc. IEEE Sensors 2011, pages 149-152, Limerick, Ireland, Oct. 28-31 2011. doi: 10.1109/ICSENS.2011.6127269. 